Technological University Dublin

ARROW@TU Dublin

\title{
Laser Induced Breakdown Spectroscopy for Quantification of Sodium and Potassium in Minced Beef: a Potential Technique for Detecting Beef Kidney Adulteration
}

\author{
Y. Dixit \\ Technological University Dublin \\ Maria Piedad Casado-Gavalda \\ Technological University Dublin, maria.casado@tudublin.ie \\ R. Cama-Moncunill \\ Technological University Dublin
}

See next page for additional authors

Follow this and additional works at: https://arrow.tudublin.ie/schfsehart

Part of the Food Science Commons

\section{Recommended Citation}

Dixit, Y. et al (2017). Laser induced breakdown spectroscopy for quantification of sodium and potassium in minced beef: a potential technique for detecting beef kidney adulteration. Analytical Methods, vol. 9 , pg. 3314-3322. doi:10.1039/C7AY00757D

This Article is brought to you for free and open access by the School of Food Science and Environmental Health at ARROW@TU Dublin. It has been accepted for inclusion in Articles by an authorized administrator of ARROW@TU

Dublin. For more information, please contact arrow.admin@tudublin.ie, aisling.coyne@tudublin.ie, gerard.connolly@tudublin.ie.

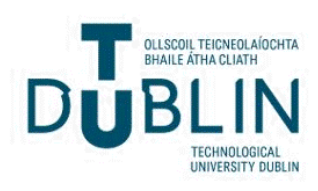




\section{Authors}

Y. Dixit, Maria Piedad Casado-Gavalda, R. Cama-Moncunill, Xavier Cama-Moncunill, Maria MarkiewiczKeszycka, Patrick Cullen, and Carl Sullivan

This article is available at ARROW@TU Dublin: https://arrow.tudublin.ie/schfsehart/291 


\section{ARTICLE}

\section{Laser Induced Breakdown Spectroscopy for quantification of sodium and potassium in minced beef: a potential technique for detecting beef kidney adulteration}

Received 00th January 20xx, Accepted 00th January 20xx DOI: $10.1039 / \times 0 \times x 00000 x$

www.rsc.org/

\section{Y. Dixit ${ }^{a}{ }^{+}$, Maria P. Casado-Gavaldaa ${ }^{a}$, R. Cama-Moncunilla ${ }^{a}$, X. Cama-Moncunilla, Maria Markiewicz-} Keszycka $^{a}$, P. J. Cullen ${ }^{\mathrm{a}, \mathrm{b}}$, Carl Sullivan ${ }^{\mathrm{a}}$

Beef is a rich source of important minerals, with potassium (K) being the most abundant mineral quantitatively except in cured meats where $\mathrm{Na}$ from the added salt predominates. This study evaluates the potential of LIBS for quantification of the sodium ( $\mathrm{Na}$ ) and potassium $(\mathrm{K})$ contents of minced beef as a potential method of detecting beef kidney adulteration. Additionally, the study aims at demonstrate the ability of LIBS to provide spatial mineral information of minced beef. A LIBS system was employed to collect spectral information of adulterated minced beef samples. Atomic absorption spectroscopy (AAS) was used to obtain reference values for $\mathrm{Na}$ and $\mathrm{K}$. The chemometric technique of partial least squares regression (PLSR) was used to build the prediction models. Spatial mineral maps of minced beef samples were generated based on the predicted percentages of $\mathrm{Na}$ and $\mathrm{K}$. The models for $\mathrm{Na}$ and $\mathrm{K}$ yielded calibration coefficients of determination $\left(R_{c}^{2}\right)$ of 0.97 and 0.91 respectively. Similarly, a good calibration model was obtained for adulteration yielding a $R_{c}^{2}$ of 0.97 . Good prediction accuracy was observed for all models. Spatial mapping provided two major advantages: (a) representative measurements of samples and (b) spatial distribution of multi-elements. The results observed illustrate the ability of LIBS combined with chemometrics as a potential monitoring tool for mineral quantification as well as adulteration detection for the meat processing industry.

\section{Introduction}

Meat and meat products are popular among consumers due to their nutritional value and taste. It is projected that world meat production will double by 2050 , with demand driven particularly in developing countries. ${ }^{1}$ Beef is a popular meat because of its flavour and nutritional quality. Minced beef is the main ingredient for products such as sausages, hamburger patties, meatballs and meat paste. ${ }^{2,3}$ Beef is a rich source of important minerals such as sodium $(\mathrm{Na})$, potassium $(\mathrm{K})$ and phosphorus $(\mathrm{P})$. Potassium is the most

a School of Food Science and Environmental Health, Dublin Institute of Technology, Dublin 1, Ireland.

bSchool of Chemical Engineering, University of New South Wales, Sydney, Australia .t Corresponding author: Y. Dixit, School of Food Science and Environmenta Health

Dublin Institute of Technology, Cathal Brugha St, Dublin 1, Ireland

Tel: +35314024543

E-mail: yash.dixit@mydit.ie abundant mineral except for cured meat where $\mathrm{Na}$ from the added salt predominates ${ }^{4}$. The importance of $\mathrm{Na}$ and $\mathrm{K}$ for humans has been extensively studied ${ }^{5-9}$; one of the basic functions of $\mathrm{Na}$ and $\mathrm{K}$ is to maintain electrolytes and fluid balance in human body. ${ }^{10}$ Declaration of mineral content in food products is a regulatory requirement as per European Union (EU) regulations and hence, mineral detection and quantification is important for complying with food regulations. ${ }^{11}$

The growing meat market provides a significant opportunity to the meat processing industry while simultaneously posing the challenge of providing safe and hygienic meat products ${ }^{1}$. One of the major issues faced by the meat processing industry is adulteration. Minced beef products have been targeted for adulteration, with cheaper substitutes such as offal. Food product authenticity is a regulatory requirement to protect consumer health, to meet the expectations of consumers and to ensure fair- 
trade. ${ }^{12,} 13$ Consumers are concerned about the composition and safety of the food they consume and particularly so with regards to meats ${ }^{14-16}$ Hence, adulteration detection in minced meat is of the highest importance in order to assure product quality and consumer protection. ${ }^{17}$

Laser-induced breakdown spectroscopy (LIBS) is an emerging technique for mineral analysis of food with potential for at-line monitoring. LIBS is a technique based on atomic emission spectroscopy (AES) in which a laser pulse ablates a small amount of material, typically a few micrograms. A plasma is formed containing a mixture of excited neutral and charged species, both in atomic and molecular form. The light emissions from these excited species as they return to their ground state are measured using spectrophotometers. ${ }^{18-20}$ LIBS provides numerous advantages as a monitoring technique such as minimal sample preparation, chemical free process, rapid detection, spatial information and portability as compared to the existing technologies of ICP-OES (Inductively coupled plasma - Optical emission spectrometry) and AAS (Atomic absorption spectroscopy). ${ }^{21-27}$ Beef and beef offal, such as kidney have different contents of $\mathrm{Na}$ and $\mathrm{K}$, which could be utilized by LIBS to develop quantification models for the same. ${ }^{4}$ Moreover, the difference in mineral composition of beef and beef kidney could be exploited to detect and quantify beef kidney adulteration. However, there are several challenges associated with LIBS when used for quantification. LIBS signal intensities are affected by the physical and chemical properties of the sample. ${ }^{28}$ Chemical composition, particle size and homogeneity of the sample surface are key factors. These factors control the interaction of the laser with the sample and hence the plasma temperature, which in turn affects the relative intensities of the emission lines due to change in the amount of ablated material $18,29,30$.

LIBS as a technology emerged from laser developments in the 1960's and first saw commercialization in metallurgy. In the field of food analysis, especially with regard to meat, LIBS is still in its infancy and only a few studies have been conducted. Andersen et. al. ${ }^{18}$ used LIBS for at-line monitoring of calcium (Ca) content in minced poultry meat. It was concluded that LIBS can separate samples according to three calcium levels: very low $(<20 \mathrm{mg} / 100 \mathrm{~g}$ Ca), intermediate (20-90 mg/100 g Ca), and high (>90 mg/100 g Ca). In a recent study; Bilge et.al. ${ }^{31}$ utilized LIBS to identify different meat species i.e. beef, pork and chicken. Meat mixtures of pork- beef and chicken-beef were also analysed. Both these studies illustrated the potential of LIBS as an effective quality-monitoring tool for the meat industry.

Chemometrics plays an important role in extracting useful information from the large spectral data sets collected during LIBS analysis ${ }^{32,33}$. It employs various multivariate techniques to perform qualitative and quantitative analysis ${ }^{34}$. Andersen et. al. ${ }^{18}$ performed partial least square (PLS) modelling to predict the calcium content of minced poultry meat. Similarly, Bilge et.al. ${ }^{31}$ performed principal component analysis (PCA) to discriminate three different meat species (beef, pork and chicken) followed by PLS modelling to predict pork and chicken adulteration in beef. Literature reveals that LIBS has not been reported to date for beef kidney detection in meat. In the current study, differences in mineral composition between beef and beef kidney is leveraged to detect and quantify adulteration. There were two main objectives of this study; (a) utilize LIBS coupled with chemometrics for quantification of $\mathrm{Na}$ and $\mathrm{K}$ in dried and compressed minced beef pellets and (b) explore the potential of LIBS as a novel technique to detect beef kidney adulteration. Additionally, the study also aims at demonstrating the ability of LIBS combined with an automated sample chamber to provide spatial information of the elements, $\mathrm{Na}$ and $\mathrm{K}$ in dried and compressed minced beef samples.

\section{Materials and methods}

The procedures explained in this section were conducted in three independent batches on random days to take into account biovariability between different animals. These batches will be referred to as batch1, batch 2 and batch 3 .

\subsection{Sample Preparation}

Fresh lean beef steaks and beef kidney weighing approximately 500 g each were purchased from a local butchers shop in Dublin city, Ireland. Beef kidney was used to vary the levels of $\mathrm{Na}$ and $\mathrm{K}$ in minced beef as well as testing the ability of LIBS for detecting offal adulteration. On the same day, the beef steaks and kidney were carefully cut in order to remove the fat portion. All samples were first cut into small cubes and minced separately using a laboratory meat blender (8011G, Waring Laboratory Science, Stamford CT, USA), which was carefully cleaned using an antibacterial washing 
liquid and dried before each use. Finally, samples were placed overnight in a hot air drying oven maintained at $105{ }^{\circ} \mathrm{C}$ using disposable aluminium dishes.

On the next day, dried samples were ground into powder form using a laboratory meat blender (8011G, Waring Laboratory Science, Stamford CT, USA) followed by sieving using a mechanical siever (VS 1000, Retsch (U.K.) Limited, Parsons lane, Hope valley, U.K.) with a 103-mesh screen for 10 minutes at $70 \mathrm{rpm}$. Forty-five samples (15 samples per batch) with varying percentages of beef kidney mixed with lean beef were prepared. Each sample comprised of approximately $400 \mathrm{mg}$ of powdered mixture of lean beef and kidney containing $0 \%, 10 \%, 20 \%, 40 \%$ and $100 \%$ of kidney in triplicates. Samples were then pelleted using a hydraulic press (GS01160, Specac Ltd., Orpington, United Kingdom) by applying a force of $98 \mathrm{kN}$ for 3 minutes. All Samples were in the form of a circular disc of $13 \mathrm{~mm}$ diameter and $4 \mathrm{~mm}$ thickness.

\subsection{Atomic absorption spectroscopy analysis}

The $\mathrm{Na}$ and $\mathrm{K}$ content of lean beef and beef kidney were determined using a flame-atomic absorption spectrophotometer (SpectrAA-50, Varian, Australia) in order to obtain reference values t. Sample preparation was carried out using the standard method of AOAC (FP-3) with slight modifications; approximately $1 \mathrm{~g}$ of powdered samples were transferred to crucibles and pre-ashed on a hot plate with the careful addition of small drops of pure nitric acid (CAS 7697-37-2, Sigma Aldrich, Inc.) to aid digestion. Once the samples were completely charred, they were transferred to a muffle furnace maintained at $550{ }^{\circ} \mathrm{C}$ for 5 hours. Ashes were then dissolved into $50 \mathrm{~mL}$ volumetric flasks with $1 \mathrm{M}$ purified nitric acid (CAS 7697-37-2, Sigma Aldrich, Inc.). A $0.1 \mathrm{ml}$ aliquot and $0.2 \mathrm{ml}$ aliquot of the resulting solution was further diluted in $25 \mathrm{ml}$ and 50 $\mathrm{ml}$ of $1 \mathrm{M}$ nitric acid and $1 \%$ cesium chloride solution to maintain the mineral concentrations within the AAS optimum measuring range for $\mathrm{Na}$ and $\mathrm{K}$ respectively. The addition of cesium chloride produces a large quantity of free electrons in the flame due to cesium's low ionization energy, which in turn suppresses the ionization of sodium and potassium and thus their mutual interferences can be neglected ${ }^{35}$. For quantification, calibration curves were obtained using standard solutions of sodium (cat. no. 05201, Sigma Aldrich, Inc.) and potassium (cat. no. 96665, Sigma Aldrich, Inc.). All samples were measured in triplicates.

\subsection{LIBS spectra acquisition}

LIBS spectra were recorded using a LIBSCAN 150 system (Applied Photonics Limited, Skipton North Yorkshire, United Kingdom) which consists of a Q-switched Nd:YAG laser (ultra, Quantel laser, 601 Haggerty Lane Bozeman, MT, USA) and a series of six spectrophotometers covering the wavelength range of 185-904 nm. The experimental setup is shown in Fig. 1. The head incorporates a miniature CCD camera and 6 lens holders which collect plasma light of different wavelength regions. The laser used for sample ablation had a pulse energy of $150 \mathrm{~mJ}$ and a pulse duration of $5 \mathrm{~ns}$ operating at $1064 \mathrm{~nm}$. A repetition rate of $1 \mathrm{~Hz}$ was employed along with a $1.27 \mu$ s gate delay and $1.1 \mathrm{~ms}$ integration time in Q-switched mode. The spectrograph was externally triggered from the laser at every pulse with a delay generator. The sample was placed at a LTSD (lens to sample distance) of approximately $80 \mathrm{~mm}$ to ensure that the laser was focussed onto the sample. Samples were measured by scanning 100 different locations in a $10 \times 10$ grid pattern with the sample moved after each shot by an automated sample chamber (XYZ-750, Applied Photonics Limited, Skipton North Yorkshire, United Kingdom) with a step size of $0.70 \mathrm{~mm}$. The surface of a kidney sample before and after the measurement is shown in Fig. 2

\subsection{Data analysis}

Data analysis was performed using R. ${ }^{36}$ The "pls" package ${ }^{37}$ was used for performing PLSR (partial least square regression) along with other in-house functions.

\subsubsection{PLSR}

In a chemometric bi-linear modelling such as partial least squares regression (PLSR), pre-processing of spectral data is the most significant step in order to remove non-linearities introduced by light scatter which can have a considerable effect on the spectra. 38 Initially, the spectra recorded for each sample at 100 different locations were subjected to mean-centering ${ }^{39}$ followed by a normalization mode proposed in a study by Castro and Pereira-Filho 40 where the raw data is divided by the highest signal in each individual spectrum. Increasing the number of spectra or shots per surface area helps to overcome the effects of material inhomogeneity. Finally, the pre-processed spectra were averaged into a single spectrum, obtaining 15 spectra per batch. Spectral data in the range of wavelengths from 567.36 to $821.169 \mathrm{~nm}$ was selected for calculations as emission peaks related to sodium ( $\mathrm{Na}$ ) 
and potassium (K) exists in this region. The processed data obtained was analysed and modelled using PLSR. PLSR is a multivariate technique that develops a linear regression model by projecting the predicted and observed variables to a new space to which $X$ and $Y$ data are transferred..$^{41}$ In this study, two different approaches were used in order to model the spectral data based on elemental concentration of $\mathrm{Na}$ and $\mathrm{K}$ or beef kidney adulteration. Since $\mathrm{Na}$ and $\mathrm{K}$ are quantitatively two of the most abundant elements in beef, they would have significant contribution to the LIBS spectra.

In order to develop the calibration models for $\mathrm{Na}$ and $\mathrm{K}$, processed data acquired for batch 1 and batch 2 (30 total spectra) were used, along with their elemental reference values extracted from AAS analysis. Similarly, for kidney adulteration, processed data acquired for batch 1 and batch 2 were used, along with their kidney percentage values calculated on a dry weight basis. Pure kidney samples were not included for developing the kidney adulteration model, as $100 \%$ kidney is not considered as an adulteration (24 total spectra). The data acquired from batch 3, along with their elemental reference values and kidney percentage values, were used as a validation sample set for the elemental models ( $\mathrm{Na}$ and $\mathrm{K}$ 15 total spectra each) and the kidney adulteration model respectively (100\% kidney not included, 12 total spectra).The method of leave-one-out was used for cross validation while developing the calibration models in order to avoid either over- or under-fitting of the models. Goodness of calibration models was evaluated by determining both root mean square error of calibration (RMSEC), root mean square error in cross validation (RMSECV) ), intercept and slope which provides information about the deviation of models from their reference values. 42 The corresponding values of both coefficients of determination in calibration $\left(R_{c}^{2}\right)$ and in cross validation $\left(R_{c v}^{2}\right)$ were also calculated in order to evaluate the goodness of fit for the models. The prediction accuracy of the developed calibration models was evaluated by calculating the root mean square error of prediction (RMSEP), corresponding coefficients of determination in prediction $\left(R_{p}^{2}\right)$ and bias values. ${ }^{43,44}$ Bias values were calculated as follows:

bias $=\left(\frac{\sum_{i=1}^{n_{v}}\left(\hat{y}_{i}-y_{i}\right)}{n_{v}}\right)$

Where, $n_{v}$ is the number of data samples for validation, $\hat{y}_{i}$ is the prediction value and $y_{i}$ is the measured value. The average differences between predicted and actual values were considered as bias.

\subsubsection{Spatial mapping}

Spatial mapping of minerals using LIBS have been reported with biological samples and infant formula. ${ }^{45-47}$ In the current study, LIBS coupled with an automated sample chamber was evaluated for spatial prediction of $\mathrm{Na}$ and $\mathrm{K}$ contents in minced beef sample pellets in order to study the elemental distribution within a sample, as well as an indication of homogeneity and therefore accuracy in the calibration models.

Quantification models obtained for $\mathrm{Na}$ and $\mathrm{K}$ were used to spatially predict $\mathrm{Na}$ and $\mathrm{K}$ content distribution in the areas analysed for batch 3. Raw data acquired from batch 3 was pre-processed by applying mean-centering followed by normalization using the individual spectral maximum, obtaining 1500 spectra with every 100 spectra corresponding to 100 shots of an individual sample. Spatial mineral maps of dried and pelleted minced beef samples were generated in $\mathrm{R}$ based on predicted values using a false colour scheme, with each colour corresponding to a different percentage of $\mathrm{Na}$ or $\mathrm{K}$.

\section{Results and Discussion}

\subsection{Atomic absorption spectroscopy analysis}

AAS was performed to determine the concentration of $\mathrm{Na}$ and $\mathrm{K}$ in lean beef and beef kidney. The accuracy of AAS results rely heavily on the calibration curve obtained using standard solutions of the desired element. Good calibrations were obtained for both $\mathrm{Na}$ and $\mathrm{K}$ in all batches with a $R_{c}^{2}$ of 0.99 . Results of AAS analysis are illustrated in Table 1. AAS results indicate that Na content in lean beef is generally lower and $\mathrm{K}$ content is generally higher than in beef kidney. One way analysis of variance (ANOVA) was performed on the $\mathrm{Na}$ levels for lean beef batches. The $\mathrm{Na}$ levels were not significantly different between these batches (Table 1). Similar analysis showed this to also be the case with regard to $\mathrm{K}$ levels for lean beef. However, with regard to beef kidney both $\mathrm{Na}$ and $\mathrm{K}$ levels respectively showed significant differences (Table 1 ). The results shown in Table 1 were in good agreement with those reported in the literature ${ }^{31}$ and were used as the reference values for the calibration and validation models. 


\section{ARTICLE}

Table 1 Sodium and potassium content in dry matter (DM) of samples determined by AAS over three independent batches.

\begin{tabular}{|c|c|c|c|c|c|}
\hline Sample & Batch & $\mathrm{Na}(\mathrm{g} / 100 \mathrm{~g} \mathrm{DM})$ & $P$ value & $\mathrm{K}(\mathrm{g} / 100 \mathrm{~g} \quad \mathrm{DM})$ & $P$ value \\
\hline $\begin{array}{l}\text { Lean } \\
\text { beef }\end{array}$ & 1 & $0.21( \pm 0.01)(0.20-0.22)$ & & $1.34( \pm 0.02)(1.31-1.36)$ & \\
\hline Powder & $\begin{array}{l}2 \\
3\end{array}$ & $\begin{array}{l}0.24( \pm 0.01)(0.23-0.25) \\
0.26( \pm 0.04)(0.23-0.30)\end{array}$ & $P>0.05$ & $\begin{array}{l}1.29( \pm 0.08)(1.22-1.38) \\
1.40( \pm 0.01)(1.39-1.41)\end{array}$ & $P>0.05$ \\
\hline $\begin{array}{l}\text { Beef } \\
\text { kidney } \\
\text { powder }\end{array}$ & $\begin{array}{l}1 \\
2 \\
3\end{array}$ & $\begin{array}{l}0.93( \pm 0.03)(0.90-0.95) \\
0.84( \pm 0.04)(0.82-0.90) \\
0.78( \pm 0.01)(0.77-0.79)\end{array}$ & $P<0.01$ & $\begin{array}{l}1.04( \pm 0.02)(1.03-1.06) \\
1.18( \pm 0.01)(1.18-1.19) \\
1.07( \pm 0.01)(1.06-1.08)\end{array}$ & $P<0.001$ \\
\hline
\end{tabular}

Standard deviation shown in brackets, preceded by the symbol $\pm(n=3)$. Numbers in brackets succeeding standard deviations correspond to the minimum and maximum values respectively.

\subsection{Spectral analysis}

samples based on $\mathrm{Na}$ and $\mathrm{K}$ contents, which are in confirmation

LIBS spectra are quite complex due to the emission of multi elements from samples. ${ }^{41}$ Fig. 3a shows the mean-centered and normalized LIBS spectra of powdered lean beef and beef kidney. Each spectrum corresponds to an average of 100 spectra collected at different locations of the pellet in order to overcome sample heterogeneity. Emission peaks related to various elements in Fig. 3a have been identified with reference to the NIST database 48 and presented in Table 2. In Fig. 3a, spectral lines related to $\mathrm{Na}$ and $\mathrm{K}$ are evident and clearly differentiates lean beef from beef kidney. $\mathrm{Na}$ and $\mathrm{K}$ exists under group 1 of the periodic table, having a single electron in their outer shell and allowing LIBS to easily excite the lone electron and subsequent detection of these elements. ${ }^{49}$ Moreover, beef and beef kidney contains high amounts of $\mathrm{Na}$ and $\mathrm{K}$ making them suitable for detection by LIBS. The relationship between the percentages of kidney adulteration in the samples is based on the difference in elemental composition, especially the difference between $\mathrm{Na}$ and $\mathrm{K}$ contents. It is evident from Fig. $3 \mathrm{~b}$ and c that the LIBS spectra clearly differentiated adulterated beef 
Table 2 Possible contributions to identified spectral peaks from various elements in the 184.547-904.123 nm region (source: NIST database ${ }^{48}$ )

\begin{tabular}{|c|c|}
\hline Central wavelength (nm) & $\begin{array}{c}\text { Possible elements as per NIST } \\
\text { database }\end{array}$ \\
\hline 279.560 & Mg II (279.552) \\
\hline 280.281 & Mg II (280.270) \\
\hline 285.231 & $\mathrm{Mg}$ I (285.212), \\
\hline 330.263 & Zn I (330.258) \\
\hline 393.386 & Ca II (393.366) \\
\hline 396.858 & Ca II (396.847) \\
\hline 422.701 & Ca I (422.673) \\
\hline 430.266 & Ca I (430.253) \\
\hline 443.491 & Ca I $(443.496)$ \\
\hline 445.497 & $\begin{array}{c}\text { Na II (445.473), Ca I (445.478), } \\
\text { K II (445.500), Fe I (445.502) }\end{array}$ \\
\hline 512.865 & Fe II (512.875) \\
\hline 516.469 & Fe I (516.455), Rb II (516.457) \\
\hline 518.396 & $\mathrm{Mg} I(518.360)$ \\
\hline 568.335 & Fe I (568.249), Na I (568.263) \\
\hline 568.945 & $\mathrm{Na} I(568.820)$ \\
\hline 588.932 & $\mathrm{Na} I$ (588.995) \\
\hline 589.645 & Na I (589.592) \\
\hline 612.251 & Ca I (612.222) \\
\hline 616.279 & Ca I (616.217) \\
\hline 643.931 & Fe I (643.876), Ca I (643.907) \\
\hline 646.347 & Ca I $(646.257)$ \\
\hline 656.348 & H I (656.290) \\
\hline 742.414 & N I (742.364) \\
\hline 744.282 & N I (744.262) \\
\hline 746.909 & N I (746.831) \\
\hline 766.458 & K I (766.489) \\
\hline 769.931 & K I (769.896) \\
\hline 777.224 & O I (777.194) \\
\hline 777.431 & O I (777.417) \\
\hline 780.010 & $\mathrm{Rb} /(780.026)$ \\
\hline 818.341 & $\mathrm{NaI}$ (818.325) \\
\hline 819.474 & $\mathrm{Na} \mathrm{I}(819.482)$ \\
\hline
\end{tabular}




\subsection{Multivariate data analysis}

PLSR has been employed as a multivariate technique to quantify various minerals in meat samples using LIBS spectral data. ${ }^{18,31}$ In the current study, PLSR was performed on pre-processed LIBS data in order to develop predictive models for $\mathrm{Na}$ and $\mathrm{K}$ contents as well as kidney adulteration in beef.

PLSR indirectly predicts the kidney adulteration in beef by exploiting the difference in the elemental composition of the samples, especially $\mathrm{Na}$ and $\mathrm{K}$ concentrations of lean beef and beef kidney due to their high content. The data from batch 3 was used as a validation set. PLSR generates linear prediction models by optimising the covariance between spectral data and the reference values (percentage of kidney, $\mathrm{Na}$ and $\mathrm{K}$ contents). In order to do so, it performs decomposition on both the spectral and reference data simultaneously. ${ }^{50,51}$

\subsubsection{Development of the calibration model}

Table 3 shows the performance summary of the developed PLSR models, containing the coefficients of determination and root mean square errors for calibration (RMSEC, $R_{c}^{2}$ ) and cross validation (RMSECV, $R_{c v}^{2}$ ), intercept and slope along with the number of latent variables (LVs) used. It also includes the coefficients of determination and root mean square errors for prediction $\left(\right.$ RMSEP,$\left.R_{p}^{2}\right)$ and calculated bias for the validation set. The model for Na showed a good fit with a $R_{c v}^{2}$ of 0.94 and the $\mathrm{K}$ model obtained a $R_{c v}^{2}$ of 0.86 . The model for kidney adulteration also showed a good fit, indicated by a high $R_{c v}^{2}$ of 0.87 . Moreover, the RMSECV for the three models were in the range of 0.03-5.27. A lower $R_{c v}^{2}$ for $\mathrm{K}$ may be attributed to the variability in emission intensities among sample replicates, along with a low variation in $\mathrm{K}$ content between lean beef and beef kidney (Fig. 1c, Table 1). At the same time, factors such as chemical composition, particle size and homogeneity of the sample surface could also have an important role in affecting the relative intensities of the emission lines. ${ }^{29}$ Furthermore, a slightly lower $R_{c v}^{2}$ and high RMSECV (5.27) for the kidney adulteration model could be related to batch-to-batch variability in the mineral composition of beef.

\section{Model validation}

Model validation is an important step, which analyses the performance of the developed PLSR model for an independent set of experiments. The primary aim of validating the model is to ensure that it will perform efficiently in the future for similar data.$^{51}$ The data obtained for batch 3 was used as a validation set. All models showed good prediction accuracy as indicated by high values of $R_{p}^{2}$ in the range of 0.87-0.94 (Table 3). Fig. 4 shows the prediction plots for $\mathrm{Na}$ and $\mathrm{K}$ contents and kidney adulteration. The model for Na showed very good performance with a $R_{p}^{2}$ of 0.94 (Fig. 4a). The prediction accuracy observed for $\mathrm{K}$ was slightly lower yielding a $R_{p}^{2}$ of 0.86 (Fig. $4 \mathrm{~b}$ ) and in accordance with the values obtained in the calibration. A lower $R_{p}^{2}$ value obtained for $\mathrm{K}$ could be attributed to the same reasons as mentioned in section 3.3.1. Good prediction accuracy was observed for kidney adulteration obtaining a $R_{p}^{2}$ of 0.87 (Fig. 4b). RMSEP values obtained for all the predictions were low and in the range of 0.04-5.28. RMSEP (5.28) and bias (-4.20) values obtained for kidney adulteration were slightly higher. As already mentioned, detection of kidney adulteration with LIBS relies on the difference in elemental composition; therefore, bio-variability within independent batches could have affected the performance of the model. Moreover, the effects due to factors such as surface homogeneity, chemical composition, particle size and LTSD cannot be neglected. Overall, the performance of the PLSR shows that the models were able to predict $\mathrm{Na}$ and $\mathrm{K}$ contents for the samples with good accuracy. The kidney adulteration model also showed promising results which could be further explored with other offal such as liver and heart in future experiments. Beef and beef liver differ in mineral content with respect to phosphorus $(\mathrm{P})$ as well as trace elements such as copper (Cu). ${ }^{4}$ In a recent study, LIBS was used for quantification of $\mathrm{Cu}$ in beef using beef liver as a natural ingredient to spike Cu levels, which could be utilized to detect liver adulteration. ${ }^{52}$

For future studies, the model performance could be further improved by controlling sampling factors such as sample homogeneity and particle size, which affect the precision and accuracy of LIBS measurements. ${ }^{19}$ Precise milling machines and freeze-drying could help in overcoming these factors. However, these procedures increase the amount of time required for sample preparation as well as possibility of contamination. Chemometric methods and mathematical corrections such as normalization could help in achieving better results with lesser time, minimum sample manipulation and minimal contamination. Moreover, LIBS need to be further explored for analysing fresh samples, increasing its 
potential to be used in an industrial enviroment. Factors such as regard.

sample geometry and matrix effects play an important role in this

Table 3. Performance of PLSR calibration models (Batch 1 and 2) and PLSR validation models (Batch 3 ) in predicting Na, $K$ contents and kidney adulteration: no. Latent variables (LVs), coefficients of determination in calibration $\left(\boldsymbol{R}_{c}^{2}\right)$, Root mean square error of calibration (RMSEC), coefficients of determination in cross-validation $\left(R_{c v}^{2}\right)$, root mean square error of cross-validation (RMSECV), intercept, slope, coefficients of determination in prediction $\left(\boldsymbol{R}_{p}^{2}\right)$, root mean square error of prediction (RMSEP) and bias. Prediction plots are shown in Fig. 4

\begin{tabular}{|c|c|c|c|c|c|c|c|c|c|c|c|c|}
\hline \multirow[t]{2}{*}{ Attribute } & \multirow[t]{2}{*}{ LVs } & \multicolumn{6}{|c|}{ Calibration (Batch 1 and 2) } & & \multicolumn{4}{|c|}{ Validation (Batch 3) } \\
\hline & & $\begin{array}{c}\text { No. of } \\
\text { samples }\end{array}$ & $R_{c}^{2}$ & $\begin{array}{c}\text { RMSEC } \\
\text { (g/100g } \\
\text { DM) }\end{array}$ & $R_{c v}^{2}$ & $\begin{array}{l}\text { RMSECV } \\
\text { (g/100g } \\
\text { DM) }\end{array}$ & Intercept & slope & $\begin{array}{c}\text { No. of } \\
\text { samples }\end{array}$ & $R_{p}^{2}$ & $\begin{array}{l}\text { RMSEP } \\
\text { (g/100g } \\
\text { DM) }\end{array}$ & Bias \\
\hline $\mathrm{Na}$ & 4 & 30 & 0.97 & 0.04 & 0.94 & 0.05 & 0.01 & 0.97 & 15 & 0.94 & 0.06 & 0.02 \\
\hline K & 3 & 30 & 0.91 & 0.03 & 0.86 & 0.03 & 0.12 & 0.91 & 15 & 0.86 & 0.04 & 0.03 \\
\hline Kidney & 4 & 24 & 0.97 & 2.72 & 0.87 & 5.27 & 0.59 & 0.97 & 12 & 0.87 & 5.28 & -4.20 \\
\hline
\end{tabular}

\section{Conclusions}

\subsubsection{Spatial mapping}

Fig. 5 illustrates the spatial mineral maps of dried and pelleted minced beef samples for batch 3. Each pixel of an image is ascribed a different colour based on the predicted percentage of $\mathrm{Na}$ or $\mathrm{K}$ at that particular location of the sample. It is evident from Fig. 5 (a) and (b) that as the percentage of kidney adulteration increases, higher values of $\mathrm{Na}$ and lower values of $\mathrm{K}$ are observed which facilitates differentiation of the kidney adulterated minced beef samples. This can be attributed to the fact that beef kidney contains higher amounts of $\mathrm{Na}$ and lower amounts of $\mathrm{K}$ in comparison to the lean beef as indicated by the AAS results. However, it can also be observed from the images that each location of an individual sample show different percentages of $\mathrm{Na}$ and $\mathrm{K}$ in Fig. 5 (a) and (b) respectively which could be related to some heterogeneity of sample pellets. Nevertheless, mineral content was fairly evenly distributed throughout the sample ensuring that samples were good representatives of their reference values and therefore ensuring robust calibration and validation models. Overall, it can be concluded from the results that spatial measurements with LIBS provides two major advantages; (a) representative measurements of heterogeneous foods such as beef and (b) spatial distribution of multi-elements in food samples.
In the present study, LIBS was successfully employed for quantification of sodium and potassium contents along with detection of beef kidney adulteration in dried and compressed minced beef samples. Moreover, LIBS was also evaluated to generate spatial mineral maps of $\mathrm{Na}$ and $\mathrm{K}$. LIBS is an emerging technique in the field of meat analysis, which provides various advantages such as minimal sample preparation, being chemical free, rapid detection, provision of spatial information and system portability.

PLSR was performed to analyse the spectral information obtained from the LIBS analysis. High values of $R_{c v}^{2}$ and low values of the corresponding RMSECV confirmed a good fit for all the models. Good prediction accuracy was observed for $\mathrm{Na}$ with a high $R_{p}^{2} 0.94$ and low RMSEP of 0.02. For K, slightly lower $R_{p}^{2}$ of 0.86 was observed which could be related to the variability in the emission intensities among sample replicates, along with a low variation in $\mathrm{K}$ content between lean beef and beef kidney. A high $R_{p}^{2}$ of 0.87 was observed for kidney adulteration; however, a slightly high RMSEP of 5.28 was also observed which could be related to the dependency of adulteration detection on bio-variability within independent batches. Moreover, factors such as surface homogeneity, chemical composition, particle size and LTSD influence LIBS performance. 
Mineral prediction maps obtained for $\mathrm{Na}$ and $\mathrm{K}$ illustrated the capability of LIBS combined with an automated sample chamber to provide spatial information as well as overcome sample heterogeneity. For future studies, improvements are required to make LIBS a suitable technique for routine analysis in an industrial environment, especially for fresh samples. Improved prediction models may be obtained by controlling sampling factors such as sample homogeneity and particle size, which affect the precision and accuracy of LIBS measurements. Adulteration detection capabilities of LIBS needs to be further explored with other offal such as liver, heart and with other meats such as pork, chicken and horsemeat. Overall, it can be concluded that LIBS combined with chemometrics demonstrates potential as a rapid monitoring tool for mineral detection and quantification as well as adulteration detection for the meat processing industry.

\section{Acknowledgements}

The authors would like to acknowledge funding from the Food Institutional Research Measure administered by the Department of Agriculture, Food and the Marine, Ireland (Grant agreement: $13 / F / 508)$.

\section{References}

1. FAO, FAO's Animal Production and Health Division: Meat \& Meat Products, http://www.fao.org/ag/againfo/themes/en/m eat/background.html, (accessed 23 JULY, 2016).

2. N. Morsy and D. W. Sun, Meat science, 2013, 93, 292-302.

3. Y. Dixit, M. P. Casado-Gavalda, R. CamaMoncunill, M. Markiewicz-Keszycka, P. Cruise, F. Jacoby, P. J. Cullen and C. Sullivan, Analytical Methods, 2016, 8, 4134-4141.

4. R. A. a. L. Lawrie, D.A., Lawrie's meat science, Woodhead Publishing Limited, 2006.

5. P. Goldstein and M. Leshem, Appetite, 2014, 79, 83-90.

6. V. Reddy, A. Sridhar, R. F. Machado and J. Chen, Journal of Integrative Medicine, 2015, 13, 1-8.
7. W. B. Farquhar, D. G. Edwards, C. T. Jurkovitz and W. S. Weintraub, Journal of the American College of Cardiology, 2015, 65, 1042-1050.

8. R. N. Ndanuko, L. C. Tapsell, K. E. Charlton, E. P. Neale, K. M. O'Donnell and M. J. Batterham, Nutrition, 2017, 33, 285290.

9. M. Singh and S. Chandorkar, Food chemistry, 2016.

10. H. R. Pohl, J. S. Wheeler and H. E. Murray, in Interrelations between Essential Metal Ions and Human Diseases, Springer, 2013, pp. 29-47.

11. European Parliament and Council, Official Journal of the European Union, 2011, L.304, 18-62.

12. G. Tøgersen, J. Arnesen, B. Nilsen and K. Hildrum, Meat science, 2003, 63, 515-523.

13. M. Kamruzzaman, D. Barbin, G. ElMasry, D.-W. Sun and P. Allen, Innovative Food Science \& Emerging Technologies, 2012, 16, 316-325.

14. N. Z. Ballin, Meat science, 2010, 86, 577587.

15. Y. Dixit, M. P. Casado-Gavalda, R. CamaMoncunill, X. Cama-Moncunill, P. J. Cullen and C. Sullivan, Journal of Near Infrared Spectroscopy, 2016, 24, 353-361.

16. D. Wu and D.-W. Sun, Innovative Food Science \& Emerging Technologies, 2013, 19, 15-28.

17. M. Kamruzzaman, D.-W. Sun, G. ElMasry and P. Allen, Talanta, 2013, 103, 130-136.

18. M.-B. S. Andersen, J. Frydenvang, P. Henckel and A. Rinnan, Food Control, 2016, 64, 226-233.

19. L. J. Radziemski and D. A. Cremers, Handbook of Laser Induced Breakdown Spectroscopy, John Wiley \& Sons, West Sussex, England, 2006.

20. J. Rakovský, P. Čermák, O. Musset and P. Veis, Spectrochimica Acta Part B: Atomic Spectroscopy, 2014, 101, 269-287.

21. J. Wang, P. Zheng, H. Liu and L. Fang, Analytical Methods, 2016, 8, 3204-3209. S. Moncayo, J. D. Rosales, R. IzquierdoHornillos, J. Anzano and J. O. Caceres, Talanta, 2016, 158, 185-191. 
23. J. Singh, R. Kumar, S. Awasthi, V. Singh and A. Rai, Food chemistry, 2016.

24. G. Bilge, B. Sezer, K. E. Eseller, H. Berberoglu, H. Koksel and I. H. Boyaci, Spectrochimica Acta Part B: Atomic Spectroscopy, 2016, 124, 74-78.

25. Z. Abdel-Salam, S. Abdel-Salam and M. Harith, Food Analytical Methods, 2017, 18.

26. S. V. Er, H. Eksi-Kocak, H. Yetim and I. H. Boyaci, Food Analytical Methods, 2016, 19.

27. V. Unnikrishnan, R. Nayak, K. Aithal, V. Kartha, C. Santhosh, G. Gupta and B. Suri, Analytical Methods, 2013, 5, 1294-1300.

28. E. C. Ferreira, E. A. Menezes, W. O. Matos, D. M. Milori, A. R. A. Nogueira and L. Martin-Neto, Food Control, 2010, 21, 1327-1330.

29. W. Lei, J. El Haddad, V. Motto-Ros, N. Gilon-Delepine, A. Stankova, Q. Ma, X. Bai, L. Zheng, H. Zeng and J. Yu, Analytical and Bioanalytical Chemistry, 2011, 400, 3303-3313.

30. F. J. Fortes, J. Moros, P. Lucena, L. M. Cabalín and J. J. Laserna, Analytical Chemistry, 2012, 85, 640-669.

31. G. Bilge, H. M. Velioglu, B. Sezer, K. E. Eseller and I. H. Boyaci, Meat science, 2016, 119, 118-122.

32. T.-L. Zhang, W. Shan, T. Hong-Sheng, W. Kang, D. Yi-Xiang and L. Hua, Chinese Journal of Analytical Chemistry, 2015, 43, 939-948.

33. J. El Haddad, L. Canioni and B. Bousquet, Spectrochimica Acta Part B: Atomic Spectroscopy, 2014, 101, 171-182.

34. K. A. Bakeev, Process analytical technology: spectroscopic tools and implementation strategies for the chemical and pharmaceutical industries, John Wiley \& Sons, 2010.

35. C. C. Pereira, H. R. S. Vitola, A. O. de Souza, A. L. Medina, M. A. Gularte and A. S. Ribeiro, Microchemical Journal, 2016, 129, 293-296.

36. R Core Team, Journal, 2014.

37. B.-H. Mevik, R. Wehrens and K. H. Liland, $R$ package version, 2013, 2.4-3.
38. A. Rinnan, F. van den Berg and S. B. Engelsen, TrAC Trends in Analytical Chemistry, 2009, 28, 1201-1222.

39. P. Pořízka, J. Klus, A. Hrdlička, J. Vrábel, P. Škarková, D. Prochazka, J. Novotný, K. Novotný and J. Kaiser, Journal of Analytical Atomic Spectrometry, 2017.

40. J. P. Castro and E. R. Pereira-Filho, Journal of Analytical Atomic Spectrometry, 2016, 31, 2005-2014.

41. G. Bilge, B. Sezer, K. E. Eseller, H. Berberoglu, A. Topcu and I. H. Boyaci, Food chemistry, 2016, 212, 183-188.

42. R. Cama-Moncunill, M. MarkiewiczKeszycka, Y. Dixit, X. Cama-Moncunill, M. P. Casado-Gavalda, P. J. Cullen and C. Sullivan, Talanta, 2016, 154, 423-430.

43. F. Tao, Y. Peng, C. L. Gomes, K. Chao and J. Qin, Journal of Food Engineering, 2015, 162, 38-47.

44. G. Romano, M. Nagle, D. Argyropoulos and J. Müller, Journal of Food Engineering, 2011, 104, 657-662.

45. Y. Gimenez, B. Busser, F. Trichard, A. Kulesza, J. Laurent, V. Zaun, F. Lux, J. Benoit, G. Panczer and P. Dugourd, Scientific Reports, 2016, 6.

46. J. Kaiser, M. Galiová, K. Novotný, R. Červenka, L. Reale, J. Novotný, M. Liška, O. Samek, V. Kanický and A. Hrdlička, Spectrochimica Acta Part B: Atomic Spectroscopy, 2009, 64, 67-73.

47. X. Cama-Moncunill, M. MarkiewiczKeszycka, Y. Dixit, R. Cama-Moncunill, M. P. Casado-Gavalda, P. J. Cullen and C. Sullivan, Food Control, 2017, 78, 304-310.

48. A. Kramida, Y. Ralchenko, J. Reader and N. A. Team, NIST Atomic Spectra Database, Version 5.3, (http://physics.nist.gov/asd).

49. E. R. Scerri, The periodic table: its story and its significance, OUP USA, 2007.

50. Y. Dixit, M. P. Casado-Gavalda, R. CamaMoncunill, X. Cama-Moncunill, F. Jacoby, P. J. Cullen and C. Sullivan, Journal of Food Engineering, 2016, 175, 58-64.

51. G. ElMasry, D.-W. Sun and P. Allen, Journal of food engineering, 2013, 117, 235-246. 
52. M. P. Casado-Gavalda, Y. Dixit, D. Geulen,

R. Cama-Moncunill, X. Cama-Moncunill,

M. Markiewicz-Keszycka, P. J. Cullen and

C. Sullivan, Talanta, 2017.

\section{Figure captions}

Fig. 1. Schematic diagram of LIBS setup.

Fig. 2. Pure kidney sample pellet (dried and compressed): (a) before LIBS analysis and (b) after LIBS analysis illustrating the $100(10 \times 10)$ craters created by the laser ablation.

Fig. 3. LIBS spectra: (a) lean beef and beef kidney, (b) sodium peaks at $568.2 \mathrm{~nm}$ and $568.8 \mathrm{~nm}$ for batch 1 (c) potassium peaks at 766.4 $\mathrm{nm}$ and $769.8 \mathrm{~nm}$ for batch 1. The upward arrow ( $\uparrow)$ indicates an increase in sodium with increase in kidney percentage and the downward arrow $(\downarrow)$ indicates a decrease in potassium with increase in kidney percentage.

Fig. 4. Prediction plots: (a) sodium content, (b) potassium content and (c) kidney adulteration for calibration and validation. $R_{p}^{2}$ indicates the coefficient of determination in prediction. Performance summary of PLSR models is shown in Table 3.

Fig. 5. Spatial mapping distribution of predicted mineral content of batch 3 pellets in triplicates (dried and compressed): (a) Na and (b) K. Each map is related to a different sample. The colour scale indicates the content of $\mathrm{Na}$ or $\mathrm{K}$ in $\mathrm{g} / 100 \mathrm{~g} \mathrm{DM}$. 


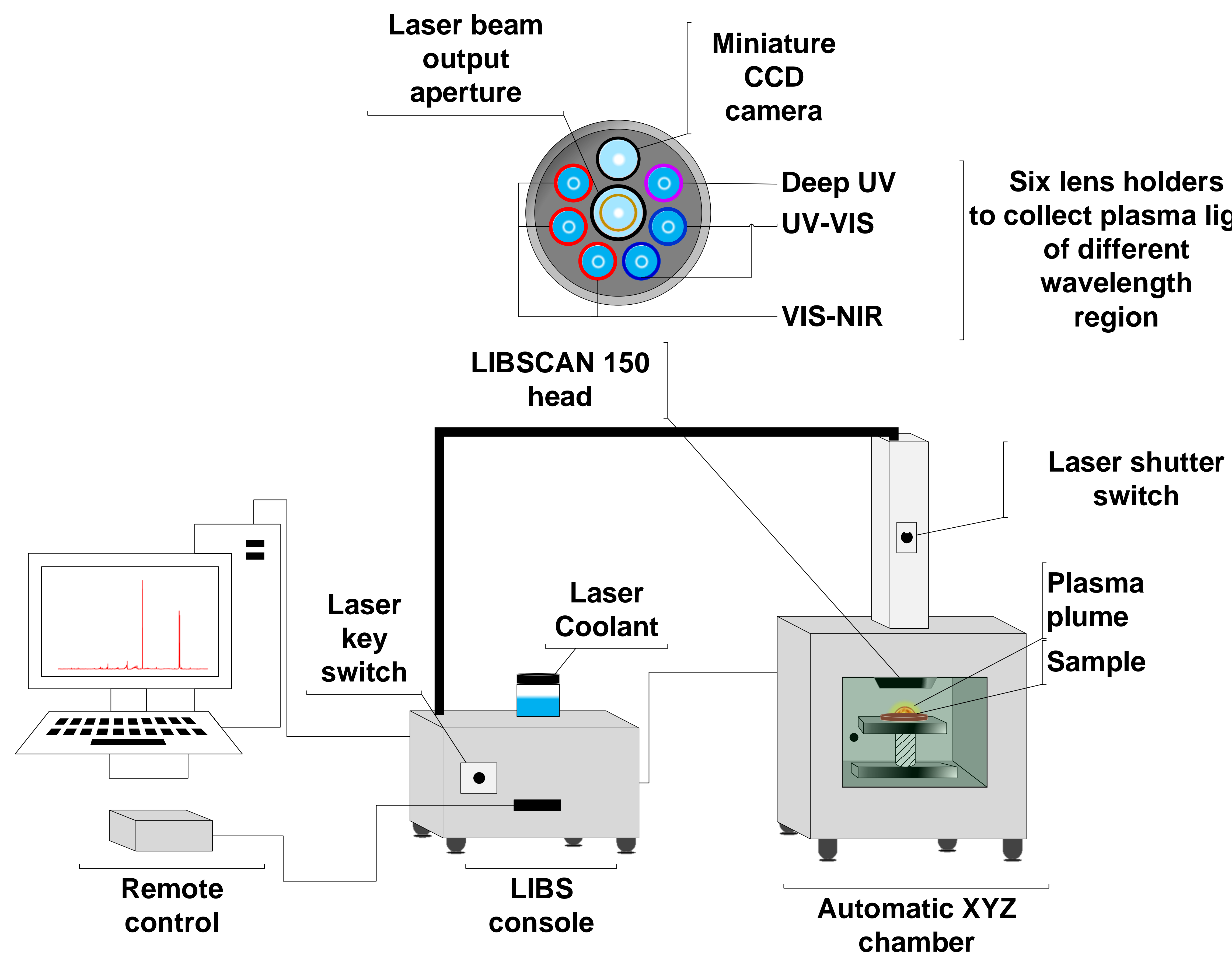




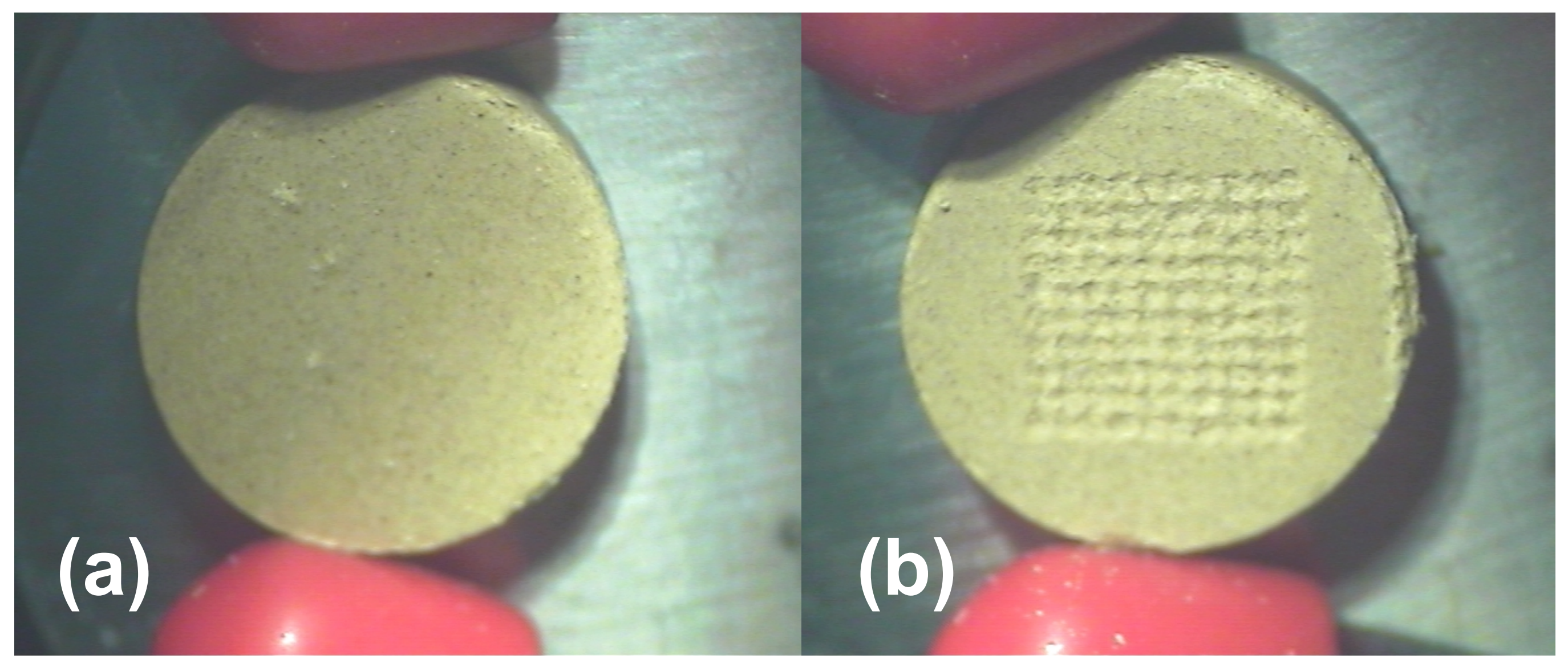




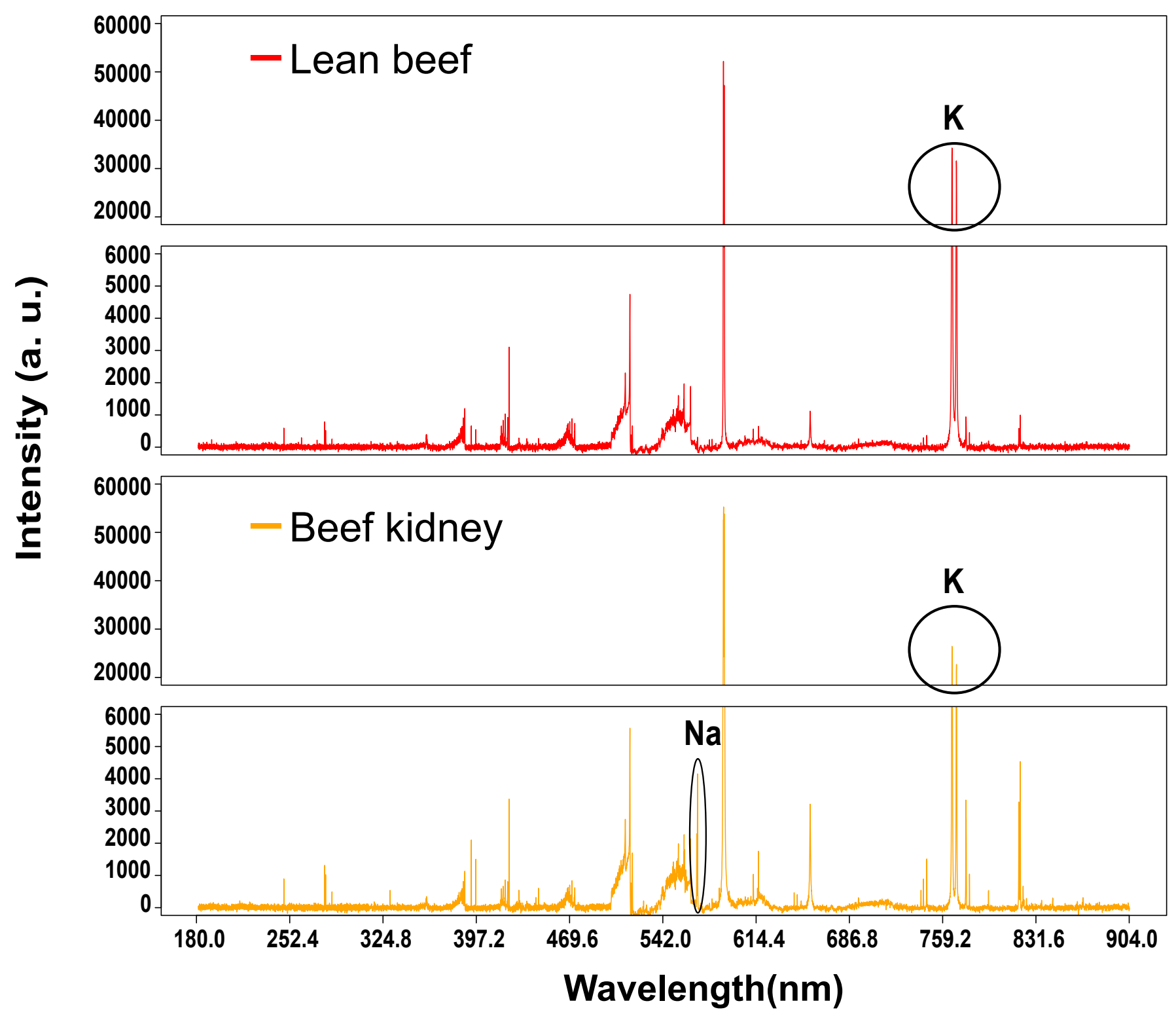

(a)

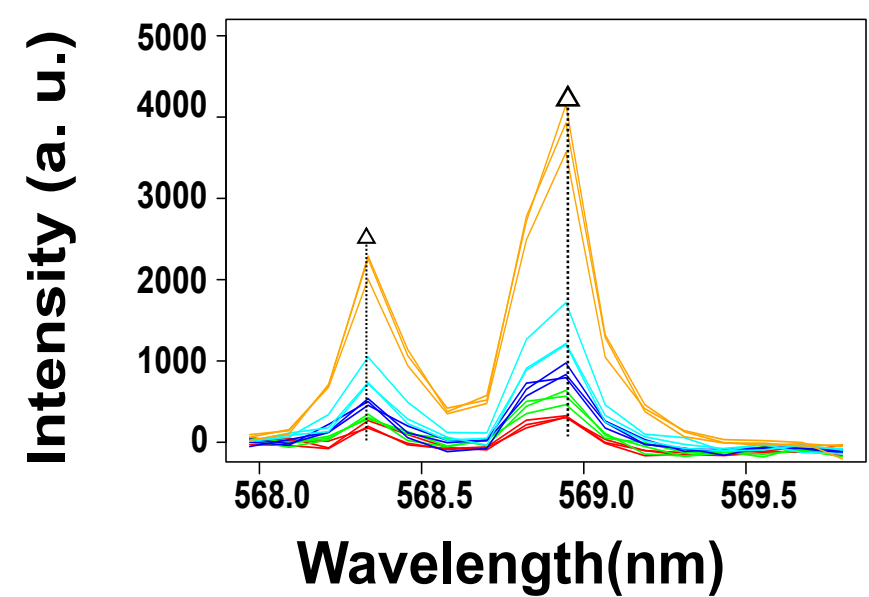

(b)

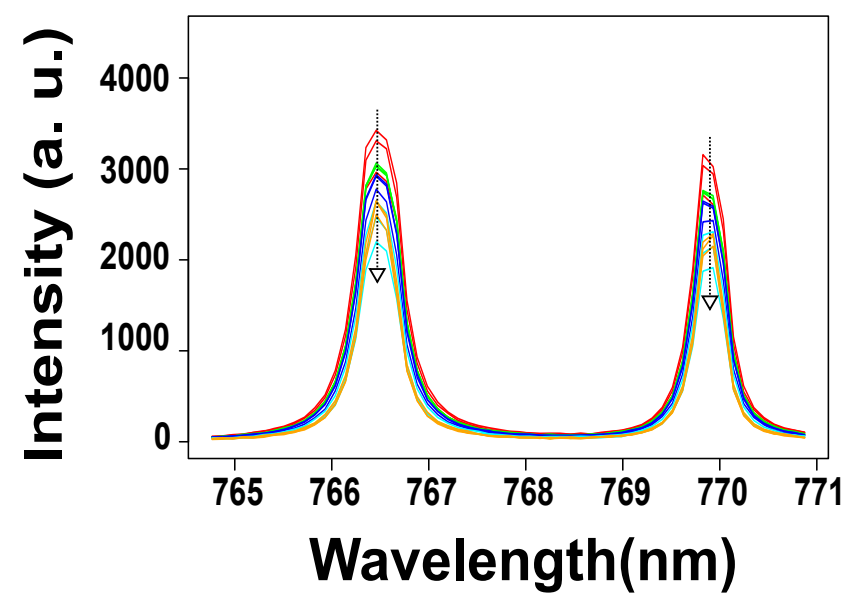

(c) 

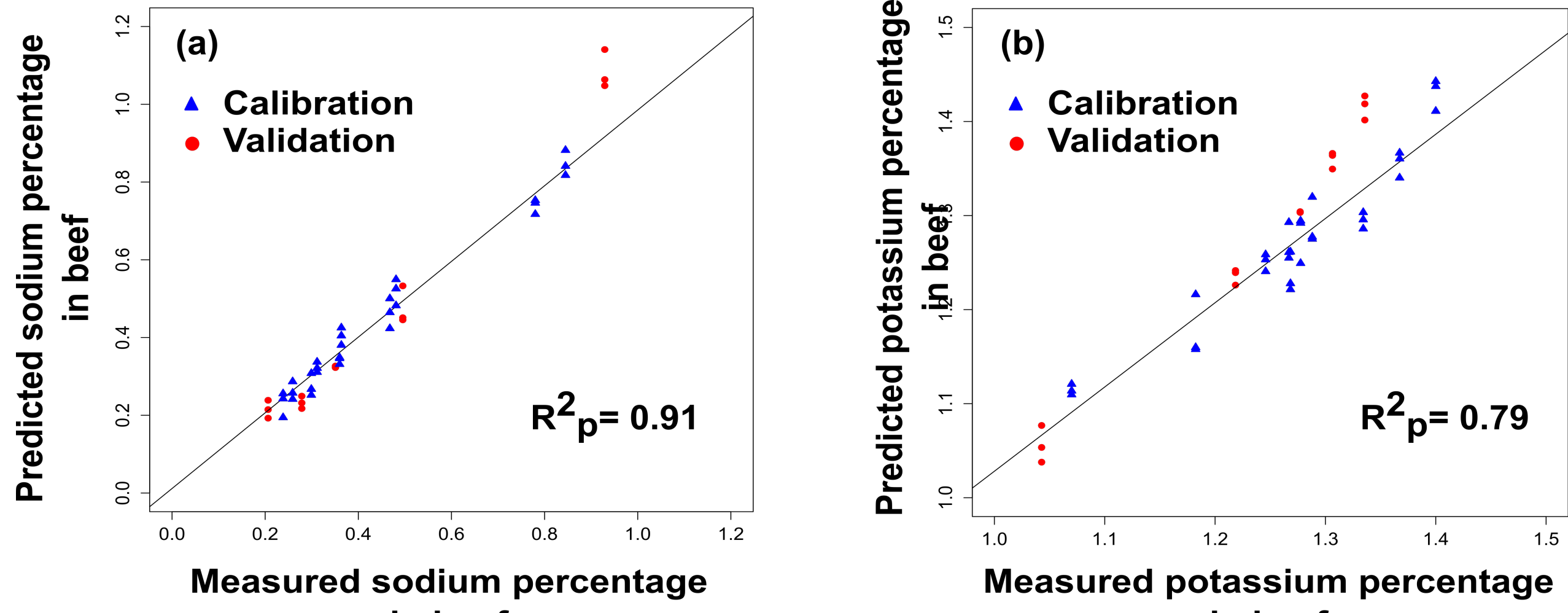
in beef in beef

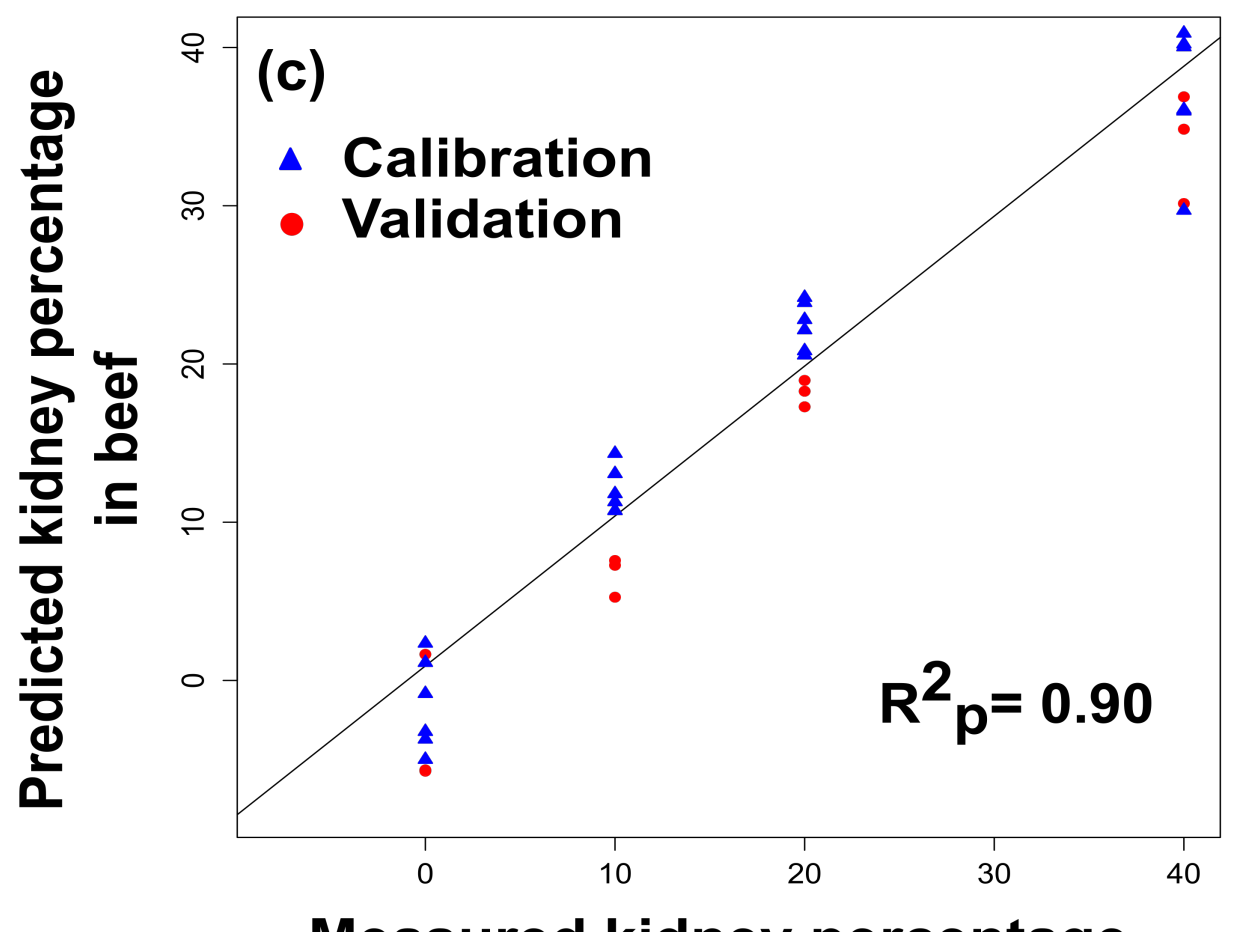

Measured kidney percentage in beef 

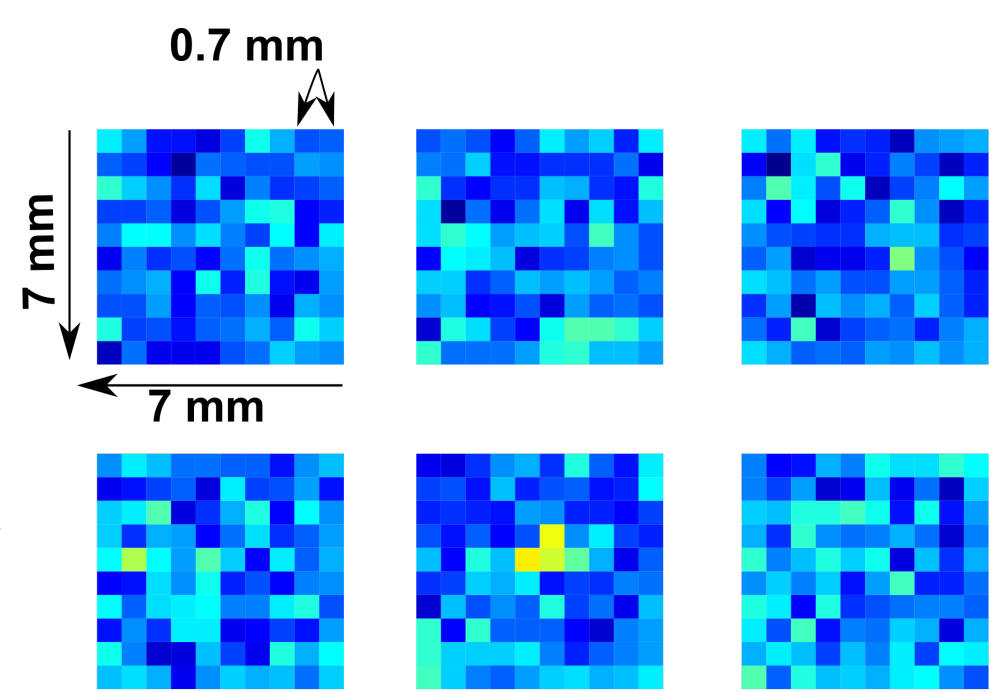

10\% kidney

20\% kidney
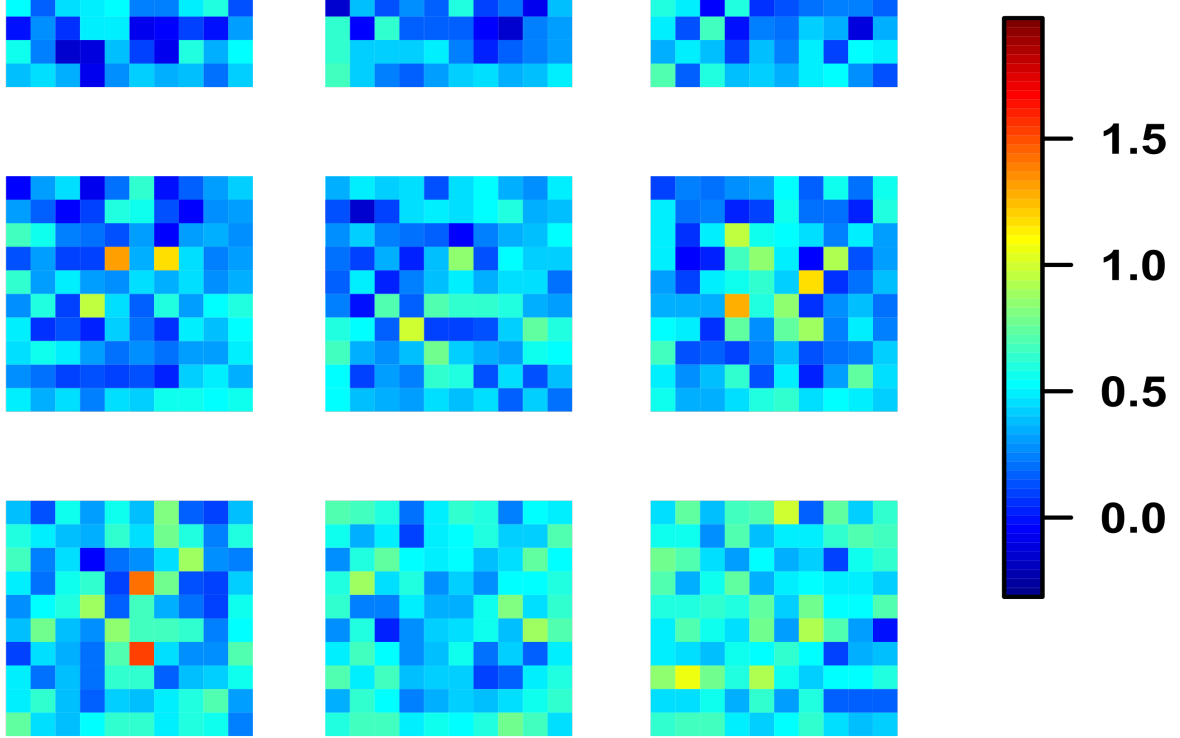

40\% kidney
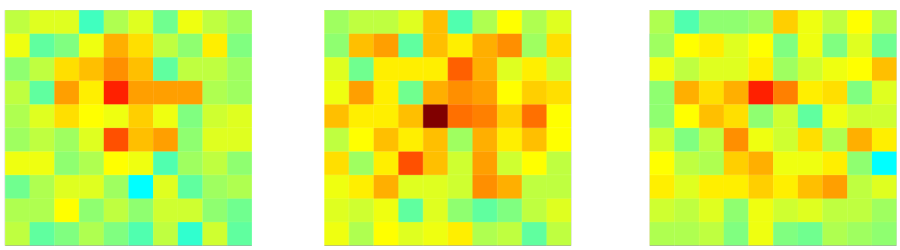

Replicate 1
Replicate 2

Replicate 3
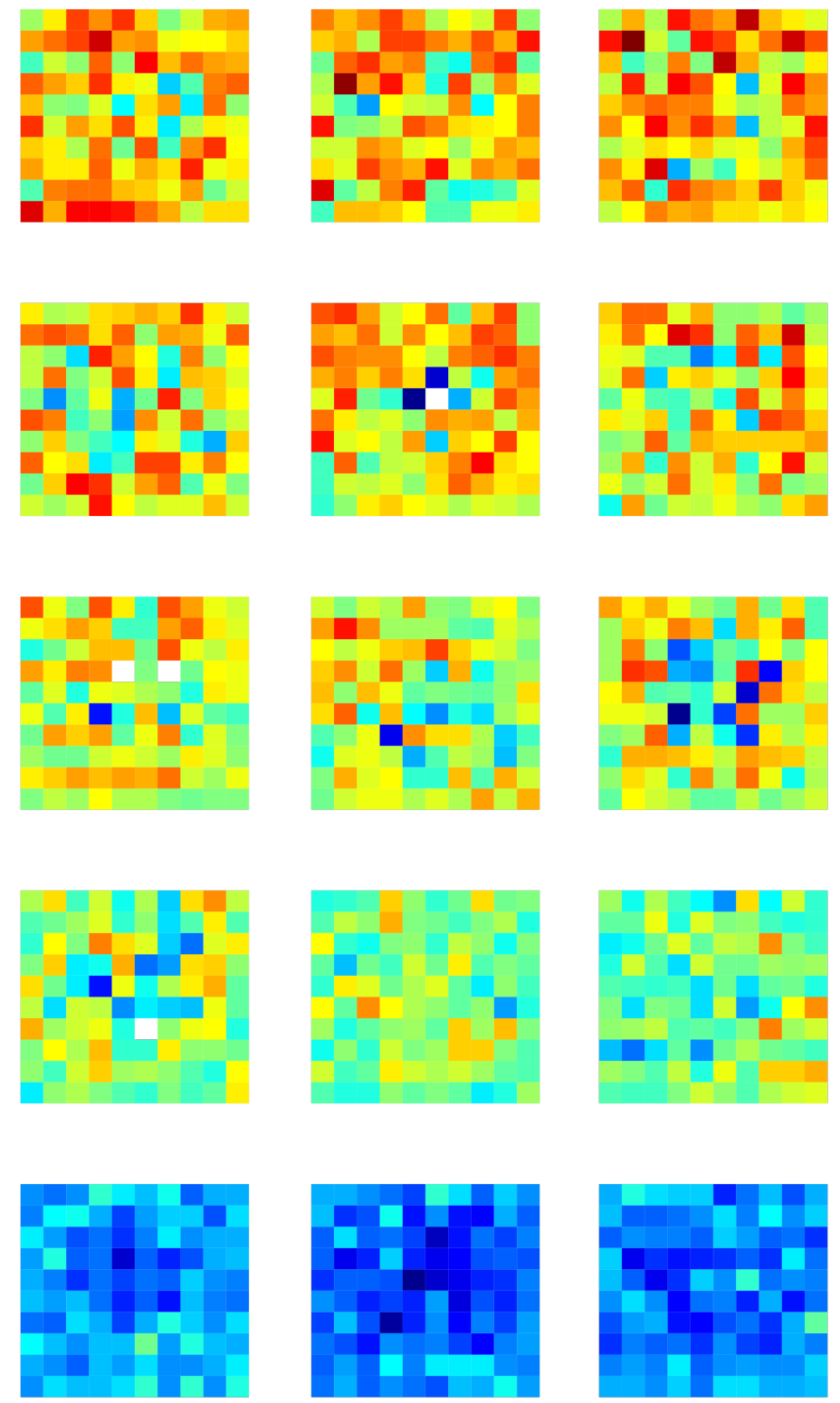

Replicate 1

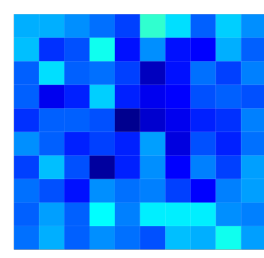

Replicate 2

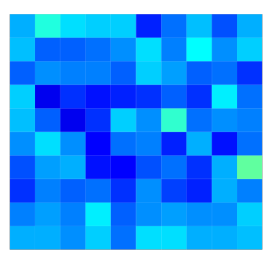

Replicate 3

(a) 


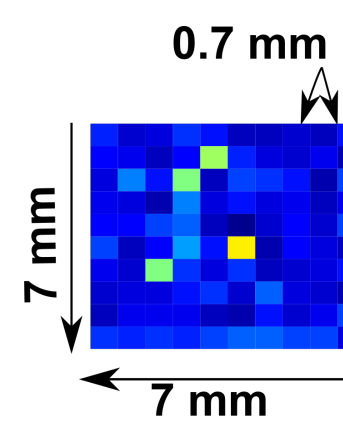

10\% kidney

20\% kidney
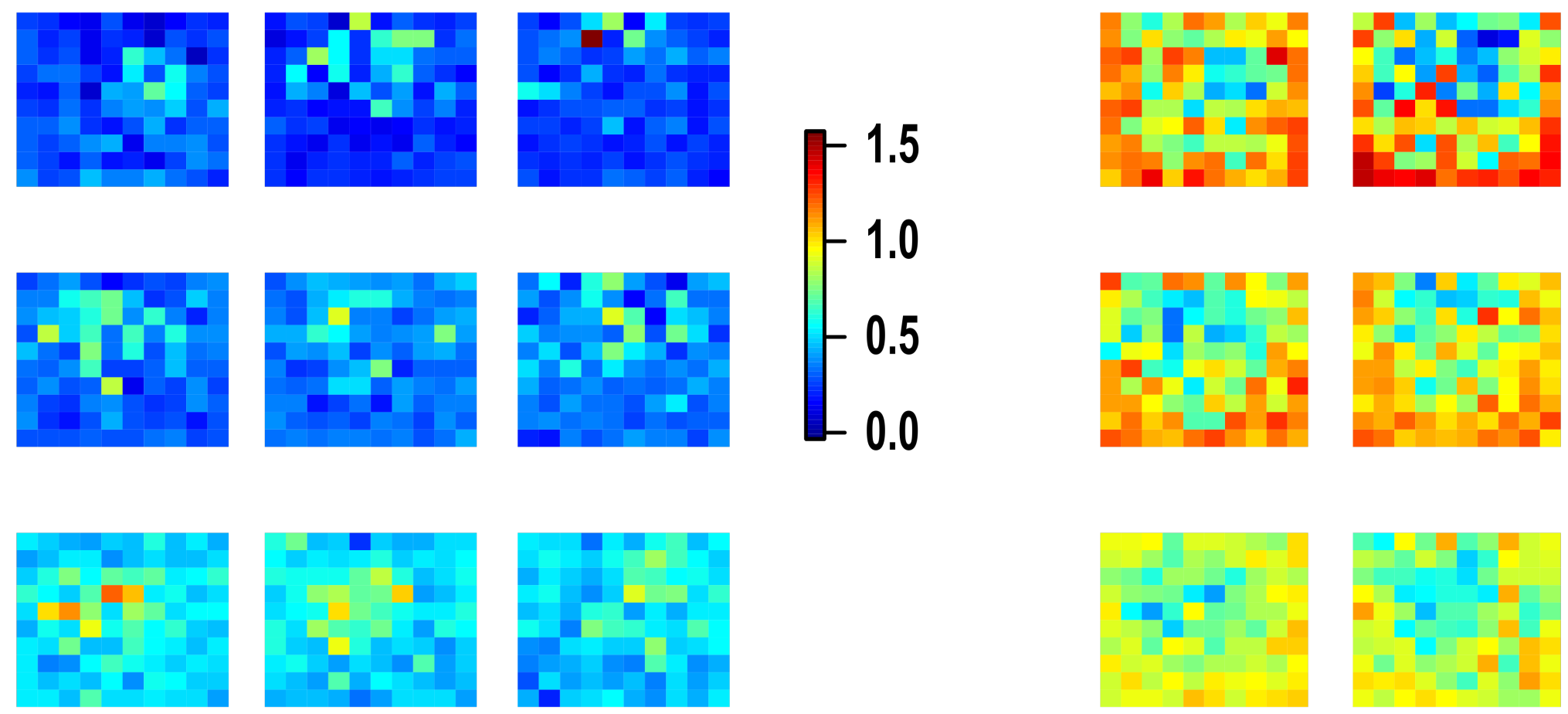

40\% kidney

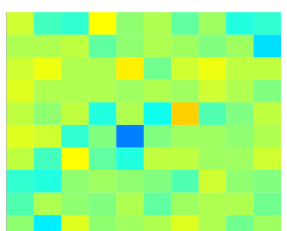

Replicate 1

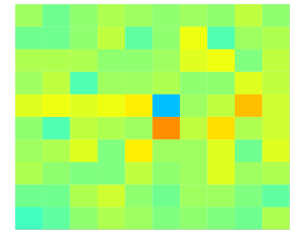

Replicate 3

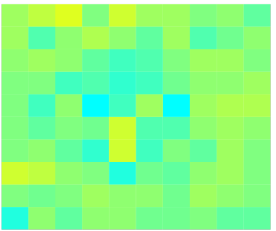

Replicate 1

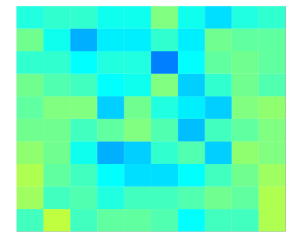

Replicate 2

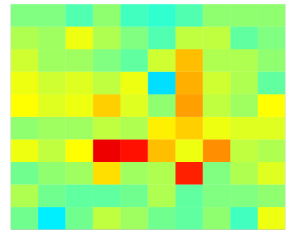

Replicate 2

(a)
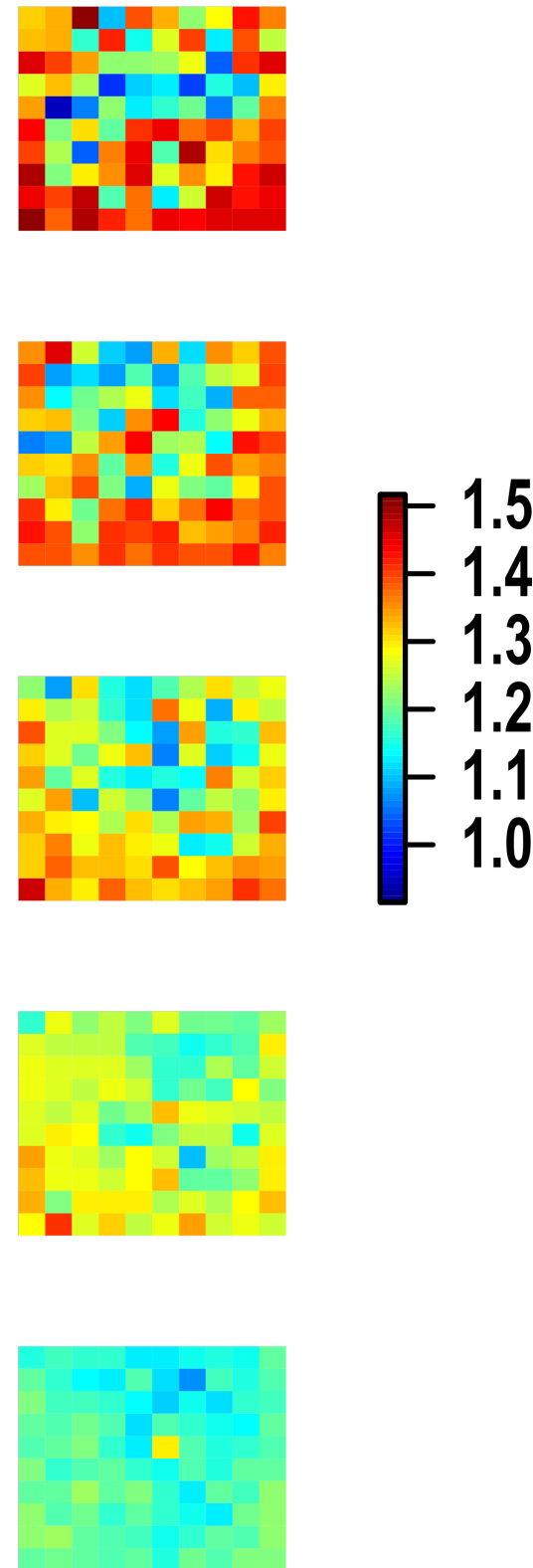

Replicate 3

(b) 

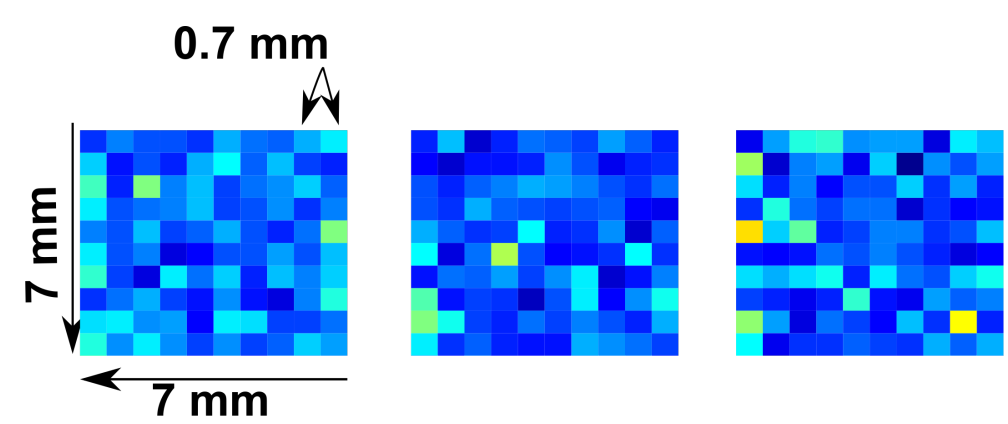

10\% kidney
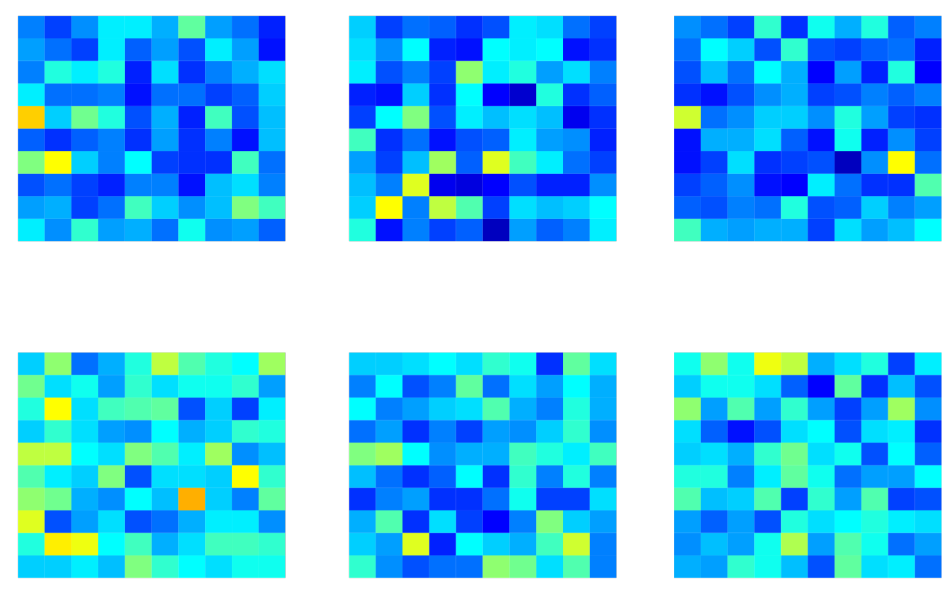

-1.0
-0.8
-0.6
-0.4
-0.2
0.0
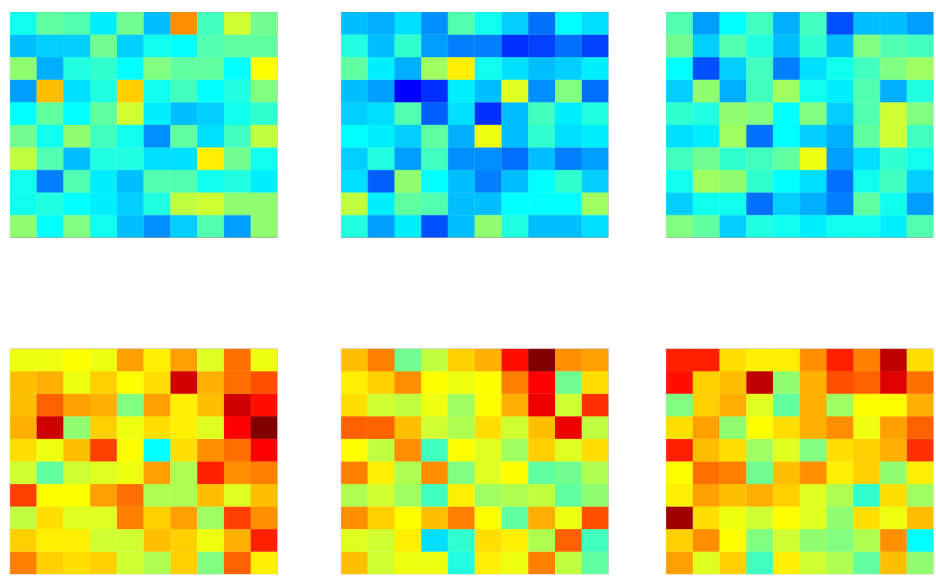

Replicate 1
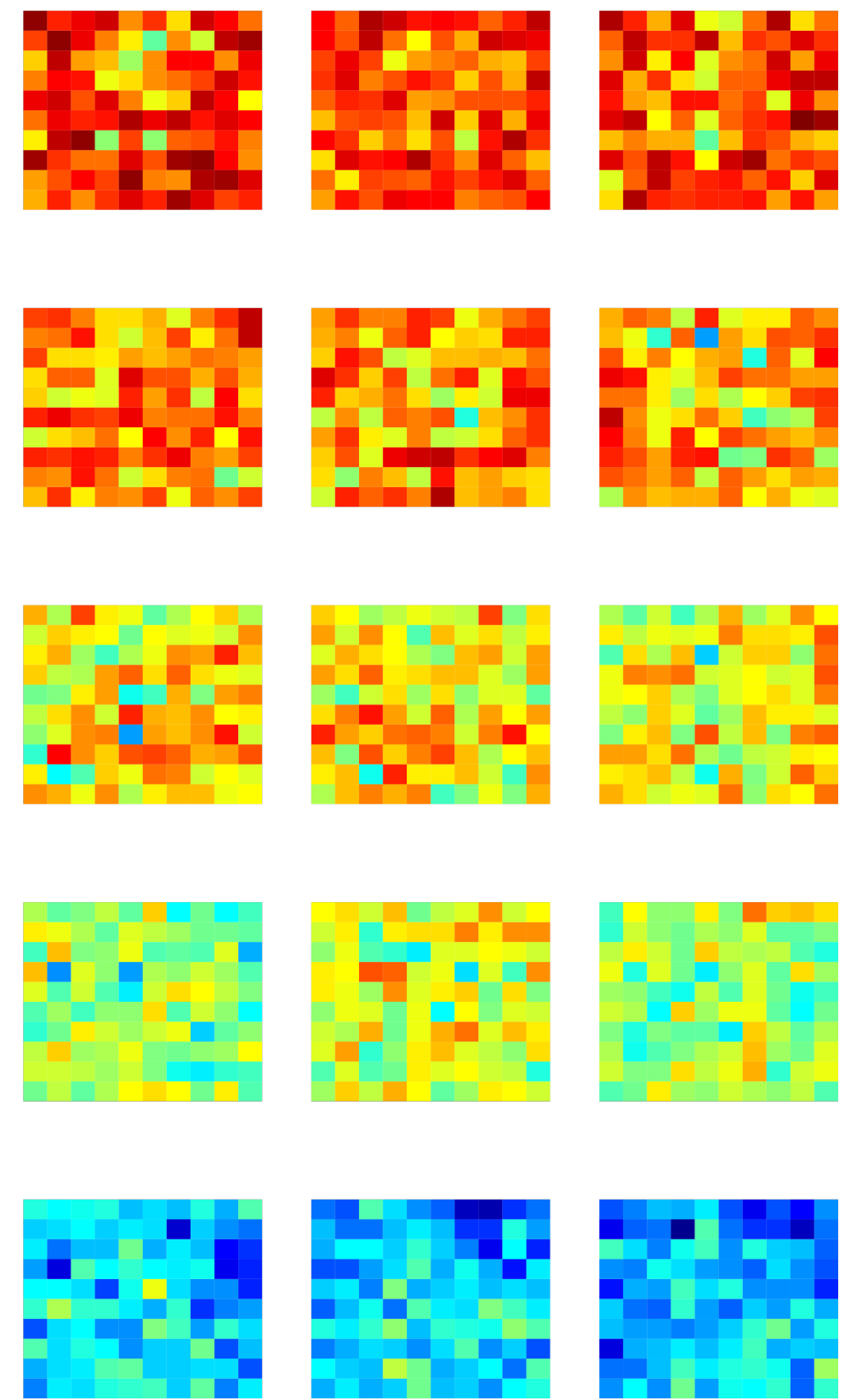

Replicate 1

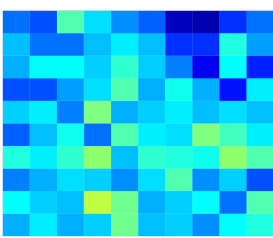

Replicate 2

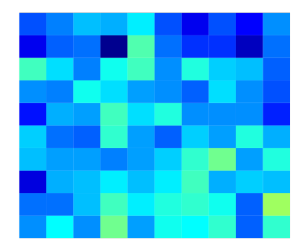

Replicate 3

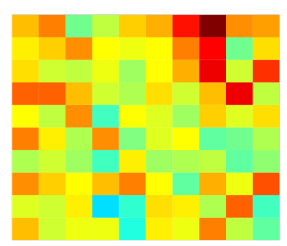

Replicate 2

(a)

(b) 